ARTíCUlO

\title{
Comportamiento espacial de la pesca artesanal en el litoral de Veracruz, México
}

\author{
Spatial behavior of artisanal fishing in Veracruz coast, Mexico \\ Pedro César Reyna-González ${ }^{1 *}$, Elizabeth Romero-Hernández ${ }^{1}$ y Juan Andrés Lorenzo-Rosas ${ }^{1}$ \\ ${ }^{1}$ Instituto Nacional de Pesca y Acuacultura-Veracruz, Av. Ejército Mexicano 106, Col. Ex Hacienda Ylang Ylang, Boca del Río, Veracruz, \\ C.P. 94298 , México \\ *Autor corresponsal: pedroreynaglez@gmail.com
}

\begin{abstract}
Artisanal fishing requires the generation of knowledge to support decision-making in the management of fisheries resources in order to achieve its sustainable use. In this sense, the delimitation of areas of fisheries exploitation has been the subject of several studies and is considered the first step in planning efforts and marine spatial planning, where the use of geographic information systems (GIS) and its application influences understanding of fisheries management. Implementing the spatial analysis, the present investigation provide an initial approach of the artisanal fishing of the 'escama marina' resource that is carried out in Veracruz coast, Mexico, considering the establishment of fishing zones and settlement of the fishing sites, the localities with the greatest resources stock in the region, the distribution of the human effort as well as the depth and operation of fishing gear. The official commercial fishing permits were reviewed for small vessels and field visits were carried out to identify main fishing cooperatives and, through the use of spatial analysis tools, established a zoning scheme divided into 3 fishing areas. Incorporating local fishermen' knowledge, we counted a total of 164 fishing sites distributed within depth strata, in which different capture systems are implemented for fishing activity. It was found that there are areas with higher concentration of fishing effort in the coast and possible social conflict zones for the fisheries resources in certain areas, which was possible to identify through the use of spatial analysis. The results of our study could become a useful tool to support the decision-making process for the design of zoning schemes and to define other management strategies for fisheries resources in Veracruz coast.
\end{abstract}

Key words: Zoning, small-scale fisheries, fishing gear, fishery conflicts, Veracruz

\begin{abstract}
Resumen.- La pesca ribereña o artesanal requiere la generación de conocimiento como soporte a la toma de decisiones en la administración de los recursos pesqueros para lograr su uso sustentable. En este sentido, la delimitación de áreas de aprovechamiento pesquero ha sido objeto de diversos estudios ya que se considera el primer paso para realizar esfuerzos de planificación y ordenamiento espacial marino, donde el uso de sistemas de información geográfica (SIG) y su aplicación influye en la comprensión del manejo de las pesquerías. Implementando el análisis espacial, el presente trabajo presenta una primera aproximación del comportamiento de la pesca ribereña del recurso escama marina que se realiza en el litoral Veracruzano, México, considerando el establecimiento de zonas de pesca y asentamiento de los sitios pesqueros, las localidades con mayor acopio del recurso en la región, la distribución del esfuerzo humano así como la profundidad y operación de las artes de pesca. Se revisaron los permisos de pesca comercial oficiales para embarcaciones menores, en campo se identificaron los principales centros de acopio; y mediante el uso de herramientas de análisis espacial se estableció un esquema de zonificación que dividió en 3 zonas de aprovechamiento pesquero al litoral Veracruzano. Integrando el conocimiento experto de pescadores locales, se contabilizaron un total de 164 sitios de captura distribuidos en diferentes estratos de profundidad, donde se implementan diversos sistemas de captura para la actividad pesquera. Se encontró que existen áreas de mayor aprovechamiento pesquero en el litoral y posibles zonas de conflicto social por las áreas de captura. Los resultados de este estudio pueden convertirse en una herramienta útil como soporte al proceso de toma de decisiones, para el diseño de esquemas de zonificación y para definir otras estrategias de manejo para los recursos pesqueros en el litoral Veracruzano.
\end{abstract}

Palabras clave: Zonificación, pesca en pequeña escala, artes de pesca, conflictos de la pesca, Veracruz

\section{INTRODUCCIÓN}

La pesca como actividad del sector productivo primario requiere de generación de conocimiento como soporte a la administración del uso de los recursos pesqueros (Arreguín-Sánchez \& Arcos-Huitrón 2011). Actualmente la principal actividad productiva en México es la agricultura seguida por la pesca (FAO \& SAGARPA 2014), por lo que históricamente y al producirse un drástico aumento del esfuerzo efectivo de pesca, se han ejercido un sin número de estrategias de manejo con el objetivo de alcanzar un enfoque ecosistémico para la pesca sustentable (Arreguín-Sánchez 2006, Ólafsdóttir \& Rafnsson 2012).
A partir de 1982, se implementaron políticas ambientales para establecer mecanismos de protección, herramientas de planeación como son los ordenamientos con procesos participativos que permitan regular los usos y las actividades productivas en ecosistemas terrestres, costeros y marinos, con el fin de lograr la protección del medio ambiente, preservación y aprovechamiento sustentable de los recursos naturales (Muñoz-Villanueva \& Acosta-Jenkins 2006, Vázquez-León 2006). Entre algunas de las políticas para el ordenamiento pesquero ha sido la implementación de permisos de pesca comercial bajo la competencia de la Comisión Nacional de Acuacultura y Pesca (CONAPESCA); 
dentro de los cuales se establecen las condiciones sustentables para el recurso pesquero, teniendo entre ellas, el esfuerzo pesquero (número de pescadores, embarcaciones y artes de pesca); la zona de pesca (límite del área de captura) así como la vigencia establecida bajo la Ley de Pesca (Ruíz-Moreno \& Mérigo-Orellana 2006, DOF 2007). Hoy en día, la Ley de Pesca y Acuacultura Sustentables precisa los lineamientos y marco legal de la pesca, en tanto que su reglamento determina regulaciones particulares, el cual tiene por objeto "garantizar la conservación, preservación y el aprovechamiento racional de los recursos pesqueros y establecer las bases para su adecuado fomento y administración" (Cruz-Ayala \& Igartúa-Calderón 2006, González-Pedrero 2006, DOF 2007¹). Fue hasta el 2011, cuando se aprobó el programa de ordenamiento ecológico marino y regional del Golfo de México y Mar Caribe creando dos áreas sujetas a ordenamiento: el área marina y el área regional, constituyéndose 57 Unidades de Gestión Ambiental (UGAs) para el estado de Veracruz con el fin de otorgar la base para la ubicación espacial de los conflictos, estrategias y lineamientos ecológicos (DOF 2012)².

En este sentido, la actividad pesquera en el estado de Veracruz es el resultado de dos fenómenos interrelacionados: su tradición histórica, donde se reporta un padrón de 42.192 pescadores, de los cuales el 36,57\% representa la población pesquera dedicada a la captura de escama ribereña (CONAPESCA 2014) ${ }^{3}$, quienes proveen del sustento a sus familias para su desarrollo sociocultural (Jiménez-Badillo 2006) y el extenso litoral costero, donde se registran cerca de 1.192 embarcaciones autorizadas para la captura de escama marina ribereña, lo que representa el 14,88\% para el estado en relación a los demás recursos pesqueros (CONAPESCA 2016) $)^{4}$. Actualmente, el grupo de escama marina lo constituyen más de 130 especies de peces que se reportan en las capturas comerciales (Quiroga-Brahms \& RomeroHernández 2013) de las cuales, más del 90\% del volumen total de capturas (15.546 t peso vivo) recae en 4 familias: Scombridae (49\%), Lutjanidae (25\%), Centropomidae (14\%) y Carangidae con el 6\% (CONAPESCA 2014) 3 . Este insumo se comercializa en fresco entero y enhielado colocando al estado como principal productor de escama marina ribereña del Golfo de México y en el quinto lugar a escala nacional (DOF 2018) ${ }^{5}$. No obstante, la industria pesquera sigue siendo incipiente, subsisten prácticas tradicionales de comercialización basadas en pequeños sitios de acopio y venta (Jiménez-Badillo 2006, CONAPESCA $2014^{3}$ ), aunado a factores como el deterioro ambiental de la zona costera, impacto de la pesca sobre el potencial reproductivo de las especies, utilización de artes de pesca de manera inadecuada, pesca furtiva, carente regulación y vigilancia insuficiente por las autoridades federales, entre otros (Contreras 2002, Jiménez-Badillo et al. 2008, QuirogaBrahms \& Romero-Hernández 2013).

Como medidas de control y manejo pesquero, las autoridades han implementado herramientas de manejo y criterios que permiten evaluar las pesquerías, tales como el cierre de zonas de pesca, delimitando zonas núcleo y amortiguamiento en áreas marinas protegidas (Beck \& Odaya 2001, Díaz de León-Corral et al. 2004, BezauryCreel 2005), el establecimiento de cuotas de captura (Ibáñez de la Calle et al. 2004), el control en las artes de pesca, temporadas de vedas, licencias y permisos (Salas et al. 2007), así como el principio precautorio (García 1994, FAO 1996); no obstante la delimitación de áreas de aprovechamiento pesquero ha sido objeto de diversos estudios ya que se considera el primer paso para una planificación y ordenamiento espacial marino (Díaz de León-Corral et al. 2006, García-Sáez 2006, Rosete et al. 2006, SEMARNAT 20126 , Espinoza-Tenorio et al. 2014) para generar información básica que permita la zonificación y caracterización espacial de la actividad pesquera (Martin 2004, Eastwood et al. 2008, Corominas-Castiñeira 2012).

La implementación de Sistemas de Información Geográfica (SIG) y su aplicación ha influido en la comprensión del manejo de las pesquerías, permitiendo la estimación de tendencias de producción pesquera por especie, área, temporada de pesca y tipo de pesquería (Ramírez-Rodríguez et al. 2004, Eastwood et al. 2008, Corominas-Castiñeira 2012). Como herramientas de planeación, el uso de SIG permite comprender la dinámica sobre la distribución de las poblaciones pesqueras e integra información sobre los factores sociales (ubicación de centros de acopio, tipos de embarcaciones, implementación de diferentes prácticas y artes de pesca, conocimiento de usuarios locales y del ambiente, entre otras) que constituyen las pesquerías para la elaboración de estrategias de manejo en un determinado espacio geográfico (Martin 2004, Eastwood et al. 2008).

${ }^{1}$ DOF. 2007. Ley general de pesca y acuacultura sustentables. Diario Oficial de la Federación, México, D.F., 24 de julio de 2007 (Última reforma publicada el 4 de junio de 2015).

${ }^{2}$ DOF. 2012. Acuerdo por el que se expide la parte marina del programa de ordenamiento ecológico marino y regional del Golfo de México y Mar Caribe y se da a conocer la parte regional del propio programa. Diario Oficial de la Federación, México, D.F., 24 de noviembre de 2012.

${ }^{3}$ CONAPESCA. 2014. Anuario estadístico de acuacultura y pesca 2014, 306 pp. <http://www.conapesca.gob.mx/work/sites/cona/dgppe/2014/ANUARIO_ ESTADISTICO_DE_ACUACULTURA_Y_PESCA_2014.pdf >

${ }^{4}$ CONAPESCA. 2016. Avisos de arribo de las capturas de escama marina en Veracruz, Oficina de pesca, sede Veracruz, México.

${ }^{5}$ DOF. 2018. Acuerdo por el que se da a conocer la actualización de la Carta Nacional Pesquera. Diario Oficial de la Federación, Ciudad de México, 11 de junio de 2018.

${ }^{6}$ SEMARNAT. 2012. Estrategia nacional para el ordenamiento ecológico del territorio en mares y costas, $33 \mathrm{pp} .<\mathrm{http}: / / \mathrm{www}$.inecc.gob.mx/descargas/ ord_ecol/est_nal_oe_mares_costas.pdf> 
El objetivo del presente trabajo fue incorporar el análisis espacial para presentar una primera aproximación del comportamiento de la pesca ribereña que se realiza en el litoral Veracruzano, considerando la información oficial disponible para el establecimiento de zonas de pesca y asentamiento de sitios pesqueros, las localidades con mayor acopio de escama marina en la región, el conocimiento experto de usuarios locales para conocer la distribución espacial de los recursos pesqueros, el funcionamiento y operación de las artes de pesca, el esfuerzo humano e identificación de áreas de mayor aprovechamiento y posibles zonas de captura de pesca que causan conflictos sociales entre los pescadores de localidades pesqueras aledañas. El enfoque implementado se considera un aporte importante para el diagnóstico pesquero en Veracruz y una propuesta de zonificación en el ordenamiento, que permitirá clarificar la dinámica de los pescadores artesanales en su territorio para fomentar estrategias metodológicas en el contexto del aprovechamiento sostenible de los recursos pesqueros en el litoral Veracruzano.

\section{MATERIALES Y MÉTODOS}

\section{ÁREA DE ESTUDIO}

El litoral Veracruzano posee una reconocida riqueza hidrológica por la extensión de su litoral de $745 \mathrm{~km}$, la superficie de lagunas costeras estimada en $172 \times 10^{3}$ ha, además de contar con 12 desembocaduras de los principales ríos del Golfo de México y amplia cobertura de cuerpos de agua dulce (Quiroga-Brahms \& Romero-Hernández 2013). La diversidad de ecosistemas y sus complejas interacciones, generan una gran riqueza de recursos que dan lugar a una importante actividad pesquera multiespecífica, la cual se distribuye geográficamente en la zona costera y aguas interiores, siendo los sistemas de captura en su mayoría de tipo artesanal (colecta manual, redes de enmalle, chinchorros, atarrayas, trampas y líneas de mano), con las especificaciones requeridas según la zona y el recurso de interés pesquero (Jiménez-Badillo 2006, Quiroga-Brahms \& Romero-Hernández 2013). De acuerdo a los registros de la Oficina de Pesca en Veracruz, en el 2016, la distribución de los recursos pesqueros a lo largo del litoral y los grupos de pescadores de escama ribereña dedicados a su extracción, se agrupan en diferentes organizaciones pesqueras en la zona norte, centro y sur del estado, respectivamente (CONAPESCA 2016) ${ }^{4}$. Cabe mencionar que la población pesquera reportada no está al 100\% activa en la pesca, ya que los pescadores suelen cambiar constantemente de permisionarios o sociedades cooperativas, así como en la captura de sus especies objetivo quedando abierta cualquier otra posibilidad que no sea la escama marina y/o pueden dedicarse a otra actividad fuera de la pesca y regresar a ella; lo último a consecuencia de las bajas capturas (temporadas), a las temporadas de nortes y otros factores externos.

\section{ZONIFICACIÓN DEL LITORAL VERACRUZANO}

En esta investigación, se revisaron 267 permisos oficiales de pesca comercial para embarcaciones menores (DOF 2002) ${ }^{7}$ emitidos por parte de la Comisión Nacional de Acuacultura y Pesca (CONAPESCA), quien es la instancia de gobierno en México encargada de regular la actividad pesquera, y en los cuales se establece la zona de pesca para la captura de escama marina, entre otros lineamientos de control pesquero. De manera conjunta, se realizó un análisis de los avisos de arribo otorgados por el sector pesquero a las diferentes oficinas de Pesca de la CONAPESCA, considerando el tipo de organización, la producción (peso en vivo en $\mathrm{kg}$ ) reportada, nombre de permisos de pesca y sitios de pesca.

Se seleccionaron las zonas de muestreo de mayor importancia en el estado por la producción pesquera reportada (Base de datos - CONAPESCA 2016) 4 . Con la información obtenida, se generó una base de datos la cual fue exportada con la plataforma SIG ArcMap Versión 10.2 (ESRI 2013) $^{8}$ para representar los límites donde opera la flota pesquera de escama ribereña, estableciendo un esquema de zonificación general con base en la ubicación de las localidades pesqueras y sus centros de acopio distribuidos en el litoral Veracruzano. Con base en este esquema de zonificación, se definieron estratos de profundidad considerando dos principales criterios: el modo en la que operan las diferentes artes de pesca y la distancia que puede recorrer una embarcación para desplazarse desde su centro de acopio y/o sitio de desembarque hacia el punto donde se realiza la captura de escama marina. Para lo anterior, se utilizó la capa de hipsometría y batimetría de INEGI y la información de límite nacional, del Geoportal de Sistema Nacional de Información de la Comisión Nacional para el Conocimiento y Uso de la Biodiversidad (CONABIO 2018) $)^{9}$, así como la base de datos con los centros de acopio geoposicionados. Con ayuda de la función de proximidad

${ }^{7}$ DOF. 2002. Acuerdo por el que se dan a conocer los formatos de Permiso de Pesca Comercial para Embarcaciones Mayores y Permiso de Pesca Comercial para Embarcaciones Menores. Diario Oficial de la Federación, México D.F., 19 de noviembre de 2002. ${ }^{8}$ ESRI. 2013. ArcMap - ArcGIS software. CD Version 10.2 para Windows.

${ }^{9} \mathrm{CONABIO}$. 2018. Hipsometría y batimetría de INEGI (1990) (escala 1:4000000) y límite nacional (escala 1:1000000) <http://www.conabio.gob.mx/ informacion/gis/> 
(buffer) en el SIG, se estableció un rango de 15 millas náuticas hacia el norte y 15 millas náuticas hacia el sur, partiendo del sitio de desembarque hacia la distancia que puede recorrer una embarcación en una y otra dirección paralela a la línea de costa, considerando ésta distancia, como el largo de cada estrato dentro de las zonas de pesca. A partir de los valores establecidos por el perfil batimétrico (CONABIO 2018) ${ }^{9}$, se definieron los límites de profundidad delimitados de manera contigua, partiendo desde la línea de costa y su parte somera, hacia el límite de la plataforma continental en su parte más profunda (parte marina), determinando así, el ancho de cada estrato a diferente intervalo de profundidad dentro de las zonas de pesca del litoral Veracruzano.

\section{UBICACIÓN ESPACIAL DE LOS SITIOS DE PESCA}

Se realizaron 627 entrevistas entre los pescadores de cada localidad pesquera del periodo correspondiente del 2013 al 2016 implementando el método de entrevista no estructurada (Dipboye 1994), la cual es una técnica de recolección de información en la investigación social y herramienta estratégica para la investigación cualitativa que permite aproximarse de forma más natural y abierta a una población de estudio, sin que se sientan irrumpidos ni examinados, de manera tal de lograr la confianza y vínculo entre el entrevistador y los entrevistados, evitando establecer previamente las preguntas ni confeccionar guiones para que los entrevistados puedan explayarse y lograr así una conversación desde sus propios intereses (Del Rincón et al. 1995, Valles 1997, Lucca-Irizarry \& Berríos-Rivera 2009). Con base en lo anterior, se obtuvo información acerca de los lugares donde los pescadores de escama ribereña llevan a cabo sus actividades (ya sea por alguna referencia espacial o punto de interés conocido por ellos o mediante el uso de GPS y registros en bitácora de pesca), la profundidad del sitio y el sistema de captura implementado de acuerdo a lo publicado en la Carta Nacional Pesquera (DOF 2000) ${ }^{10}$. La información obtenida fue integrada en una base de datos de Excel (Microsoft 2010) ${ }^{11}$ y exportada mediante la plataforma de SIG, para representar espacialmente la ubicación de los sitios y las artes de pesca distribuidas en los diferentes estratos de profundidad dentro de las zonas de pesca del litoral Veracruzano. Para resaltar la importancia de cada estrato donde se concentra la mayor actividad pesquera, se utilizó la escala cualitativa propuesta por Reyna-González et al. (2014), basada en indicadores de clasificación por escala de color propuesta por TNC (TNC 2005), para representar de manera teórica, la relevancia que posee cada estrato de profundidad (de mayor a menor importancia) con relación a las diferentes artes de pesca implementadas para la captura de escama marina en el litoral Veracruzano.

\section{Resultados}

\section{ZONIFICACIÓN DEL LITORAL VERACRUZANO}

Derivado del análisis de la información que se encuentra en los permisos de pesca comercial para la captura de escama marina, se identificaron 17 tipos de organizaciones pesqueras (Fig. 1), distribuidas en 9 principales localidades de importancia pesquera, las cuales acopian la mayor producción de escama marina, y que de acuerdo a las zonas de captura reportadas por los pescadores, se identifican

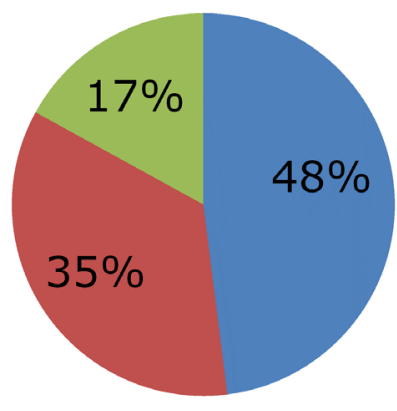

- Permisionarios

Cooperativas pesqueras

Otras (SCP, SSS, SCPP, CPA SCPP Y SERV)

Figura 1. Porcentaje de organizaciones pesqueras identificadas en el litoral Veracruzano. SCP: Sociedades Cooperativas Pesqueras, SSS: Sociedades Sociales y Servicios, SCPP: Sociedades Cooperativas de Producción Pesquera, CPA: Cooperativas Pesqueras y Acuícolas, SCPP Y SERV (Sociedades Cooperativas de Producción Pesquera y Servicios) / Fishing organizations percentage identified in Veracruz coast

\footnotetext{
${ }^{10}$ DOF. 2000. Carta nacional pesquera. Tercera sección - sistema de captura. Diario Oficial de la Federación, México D.F., 28 de agosto de 2000.

${ }^{11}$ Microsoft Office 2010. Microsoft Excel Version 2010. Copyright C Microsoft Corporation. All rights reserved.
} 
3 zonas de aprovechamiento pesquero: zona norte (que abarca desde la comunidad de Barra de Cazones hasta municipio de Vega de Alatorre), la zona centro (desde la comunidad de Chachalacas al municipio de Lerdo de Tejada) y la zona sur (a partir de Lerdo hasta Agua Dulce) en el litoral Veracruzano. Se identificaron 2 zonas pesqueras de conflicto, 1) en la localidad de Tecolutla donde se practica la pesca comercial de escama por pescadores nativos y otros tantos que se desplazan de la localidad de Casitas, teniendo estos últimos otra zona de pesca en la parte sur del litoral, y 2) entre las localidades de Alvarado y Punta Puntilla durante la temporada de pesca del róbalo (Centropomus spp.) debido a que es un lugar de reproducción para la especie (Fig. 2). De acuerdo con los permisos oficiales expedidos por CONAPESCA, no se establece una regulación ni delimitación espacial sobre la distancia y profundidad donde es permitida la pesca de escama ribereña, ya que sólo menciona como criterio que la zona de pesca sea dentro del litoral o rivera del Golfo de México, identificándose diversas artes de pesca oficiales para realizar esta actividad: cordeles, redes agalleras, redes chinchorro, red tendal, red atarralla, red de enmalle, red trasmallo, línea de cala, líneas de palangres, línea de vara, línea de mano, línea cimbra, línea de curricán y cordeles con anzuelo. Por lo anterior, se establecieron 65 estratos clasificados en 5 categorías (A: 0 a -20 m; B: -20 a -40 m; C: -40 a -100 m; D: -100 a -200 m y E: -200 a $-1000 \mathrm{~m}$ ), correspondiente a los intervalos de profundidad de la batimetría y distribuidos de la siguiente manera: 25 estratos para la zona norte, 20 para la zona centro y 20 para zona sur (Fig. 3). Estos estratos poseen diferente forma y extensión, debido a las características geomorfológicas presentes dentro y a lo largo de la plataforma continental del litoral Veracruzano.

\section{UBICACIÓN ESPACIAL dE LOS SITIOS DE PESCA}

Derivado del análisis de las encuestas realizadas, se contabilizaron un total de 164 sitios de pesca (Tabla 1), distribuidos en los diferentes estratos de profundidad de las 3 zonas de aprovechamiento pesquero. Los resultados muestran que en estos sitios, se implementan 4 principales artes de pesca especializadas para cada especie objetivo (Tabla 2); por lo tanto, los sitios de pesca ubicados espacialmente, representan las áreas de mayor

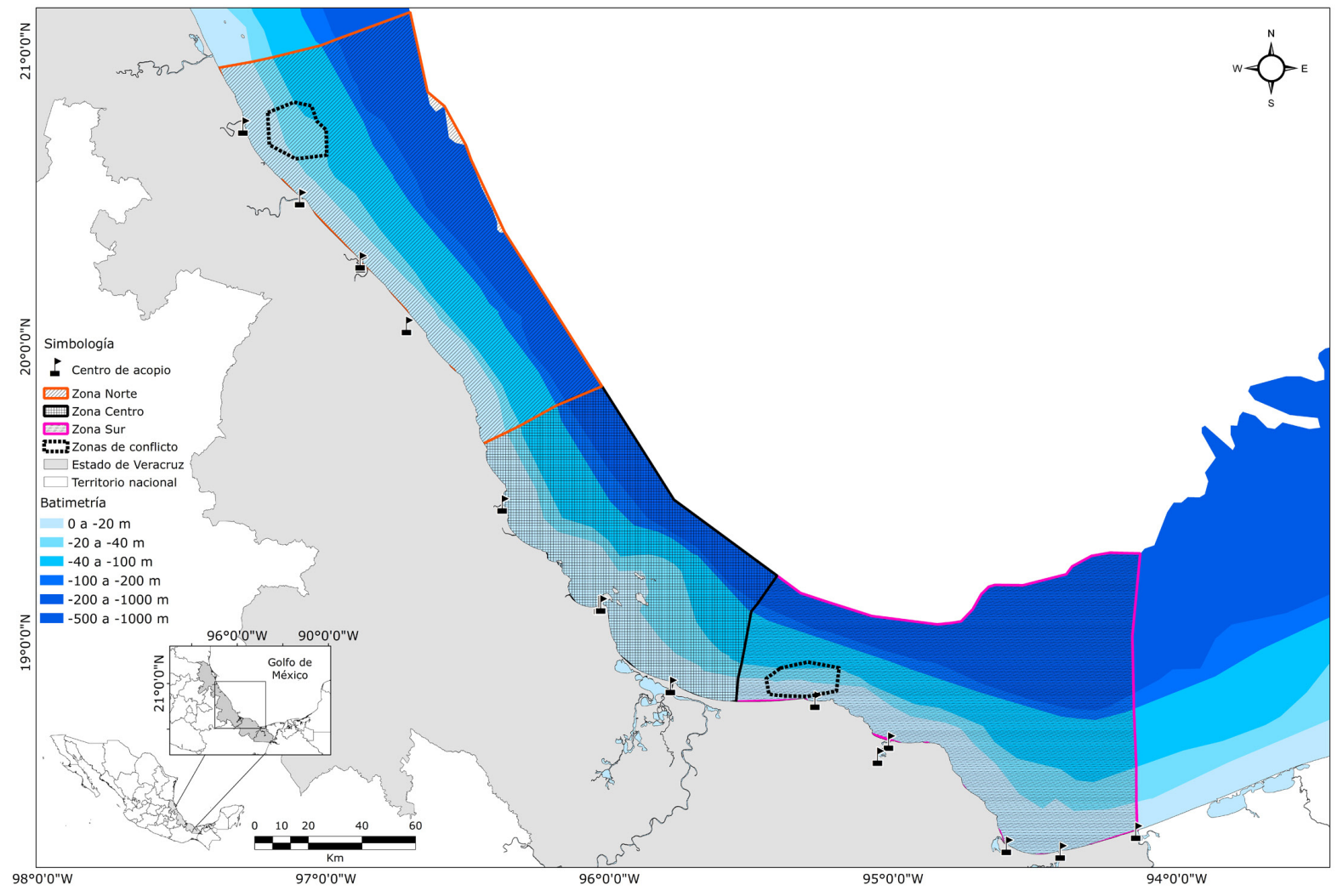

Figura 2. Esquema de zonificación que divide al litoral Veracruzano en tres áreas de aprovechamiento pesquero / Veracruz coast's zoning scheme that divides in three fishing exploitation areas 


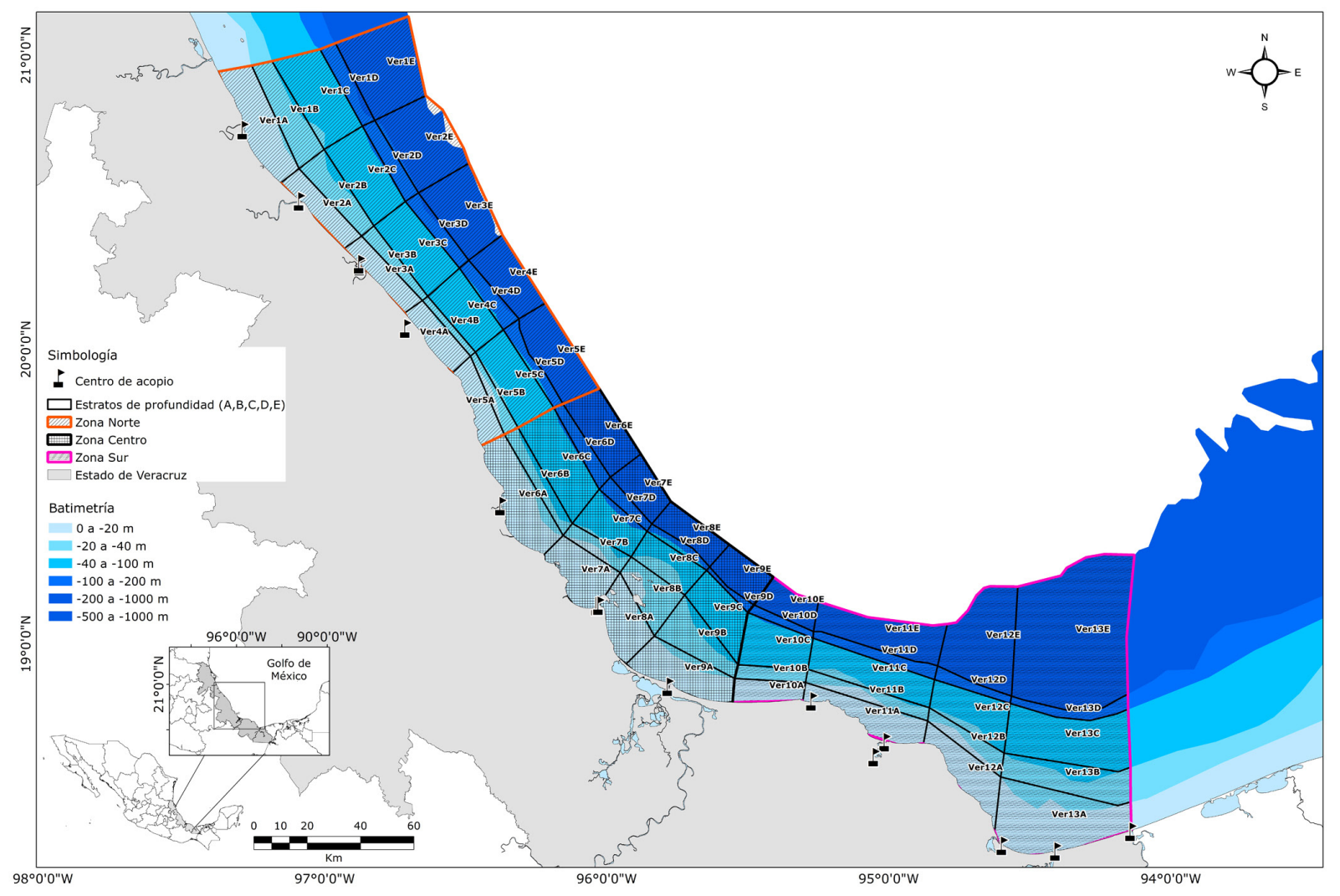

Figura 3. Estratos de profundidad distribuidos dentro del litoral Veracruzano / Depth strata distributed within Veracruz coast

Tabla 1. Número total de sitios de pesca ubicados espacialmente dentro los estratos de profundidad en el litoral Veracruzano / Total number of fishing sites spatially located within the depth strata in Veracruz coast

\begin{tabular}{lllccccc}
\hline \multirow{2}{*}{$\begin{array}{c}\text { Zona de } \\
\text { muestreo }\end{array}$} & Localidades pesqueras & \multicolumn{5}{c}{ Estratos de profundidad } \\
\cline { 3 - 7 } & & Estrato A & Estrato B & Estrato C & Estrato D & Estrato E & Total \\
\hline \multirow{2}{*}{ Norte } & Barra de Cazones & 13 & 1 & 7 & 0 & 0 & 21 \\
& Tecolutla & 9 & 13 & 19 & 0 & 2 & 43 \\
& Casitas & 0 & 1 & 3 & 0 & 0 & 4 \\
\multirow{5}{*}{ Centro } & Chachalacas & 2 & 0 & 0 & 0 & 0 & 2 \\
& Antón Lizardo & 8 & 23 & 1 & 0 & 0 & 32 \\
& Alvarado & 11 & 27 & 0 & 0 & 0 & 38 \\
& Allende & 1 & 5 & 5 & 0 & 0 & 11 \\
& Salinas Roca Partida & 11 & 1 & 18 & 2 & 1 & 33 \\
& Barra de Sontecomapan & 0 & 1 & 0 & 0 & 0 & 1 \\
\hline
\end{tabular}

Tabla 2. Artes de pesca utilizadas en las diferentes localidades pesqueras para la captura del recurso escama marina / Fishing gears used in different fishing locations to capture the 'escama marina' resource

\begin{tabular}{|c|c|c|c|c|c|c|c|c|c|}
\hline \multirow[b]{2}{*}{ Artes de pesca } & \multicolumn{9}{|c|}{ Localidades pesqueras } \\
\hline & $\begin{array}{l}\text { Barra de } \\
\text { Cazones }\end{array}$ & Tecolutla & Casitas & Chachalacas & $\begin{array}{c}\text { Antón } \\
\text { Lizardo }\end{array}$ & Alvarado & Allende & $\begin{array}{c}\text { Salinas } \\
\text { Roca } \\
\text { Partida }\end{array}$ & $\begin{array}{c}\text { Barra de } \\
\text { Sontecomapan }\end{array}$ \\
\hline Redes & $\mathrm{x}$ & $\mathrm{x}$ & & $\mathrm{x}$ & $\mathrm{x}$ & $\mathrm{x}$ & $\mathrm{x}$ & $\mathrm{x}$ & \\
\hline Curricán & & $\mathrm{x}$ & & & $\mathrm{x}$ & $\mathrm{x}$ & $\mathrm{x}$ & $\mathrm{x}$ & \\
\hline Línea de mano & $\mathrm{x}$ & $\mathrm{x}$ & $\mathrm{x}$ & & $\mathrm{x}$ & $\mathrm{x}$ & $\mathrm{x}$ & $\mathrm{x}$ & \\
\hline Palangre & $\mathrm{x}$ & $\mathrm{x}$ & $\mathrm{x}$ & & $\mathrm{x}$ & & $\mathrm{x}$ & $\mathrm{x}$ & $\mathrm{x}$ \\
\hline
\end{tabular}


aprovechamiento pesquero en el litoral Veracruzano. Los resultados obtenidos de las entrevistas muestran que existe una zonificación de carácter batimétrico con base al arte de pesca utilizado, siendo las redes las mayormente dominantes en la franja costera y zonas más someras ( 0 a $-20 \mathrm{~m})$, y la línea de mano, curricán y el palangre, implementados en las zonas más alejadas de la línea de costa, en donde opera en rangos de -40 hasta $-1000 \mathrm{~m}$ de profundidad (Fig. 4).

En cuanto al valor de importancia de los estratos de pesca, se presenta un patrón de concordancia entre la profundidad y el arte de pesca utilizada. Los resultados obtenidos muestran que, dentro de las 3 grandes zonas de aprovechamiento pesquero, hay estratos y zonas específicas en donde se concentra una mayor cantidad de sitios de pesca con respecto a otras, así como estratos en los cuales no se tuvieron registro de la actividad pesquera en estas zonas. En el caso de las redes, son utilizadas en las profundidades más bajas (entre -2 a -15 m) y en lugares más cercanos a la costa. Este arte va desde muy específico, como la red robalera, red sierra, red chopera las cuales van dirigida como su nombre lo indica a la captura de especies como el róbalo (Centropomus undecimalis y Centropomus poeyi), sierra (Scomberomorus maculatus) y chopa (Lobotes surinamensis) respectivamente; y multiespecíficas, las cuales pueden capturar especies como jurel (Caranx hippos), pargo (Lutjanus argentiventris) y otras especies de importancia comercial. Los resultados obtenidos (Fig. 5a) mostraron que los estratos de la categoría A $(0$ a -20 m) de profundidad en la zona norte y centro presentan los valores de importancia alto y medio de acuerdo con el arte de pesca (redes), ubicadas en estos sitios.

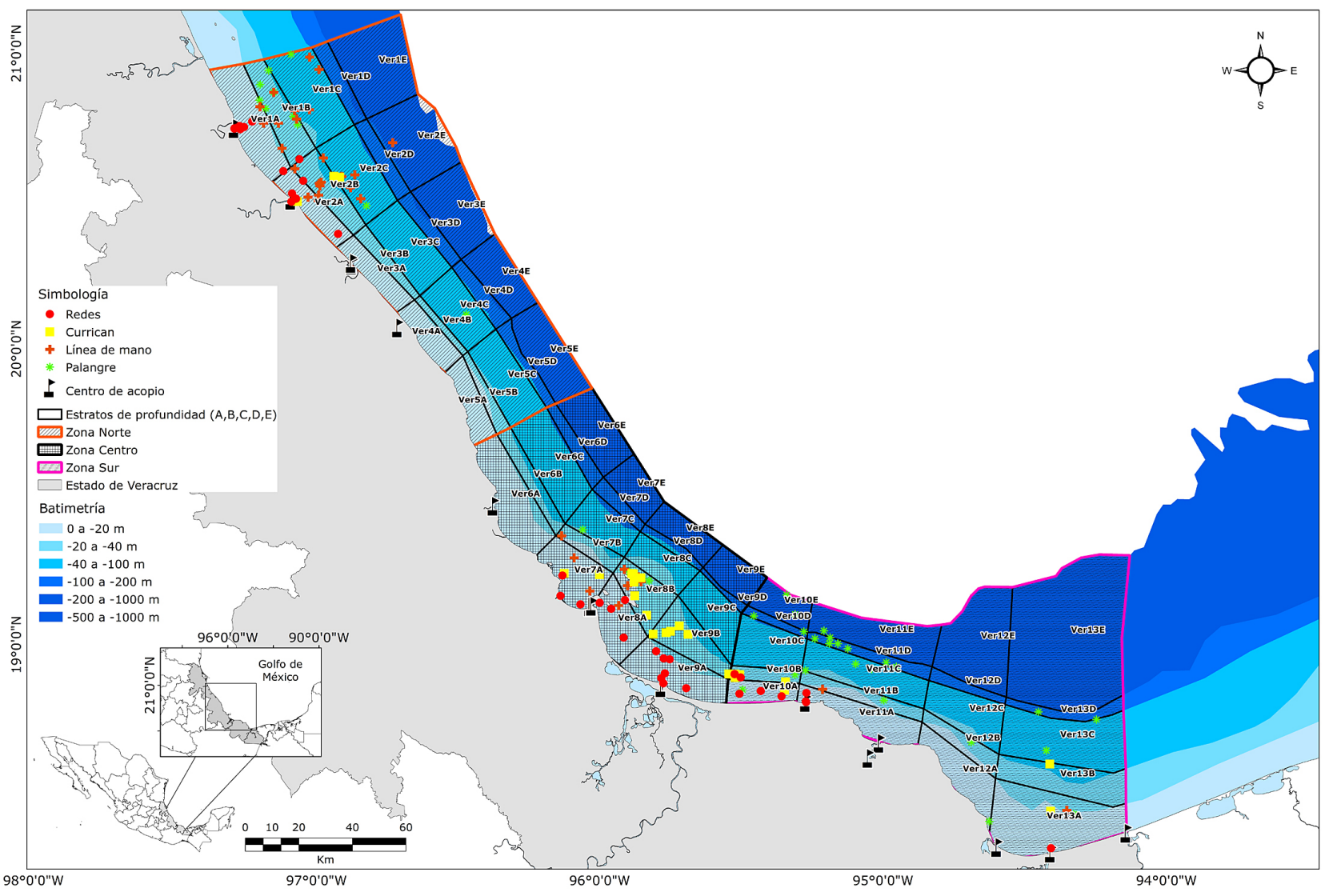

Figura 4. Distribución espacial de los sitios y artes de pesca implementadas para la captura del recurso escama marina dentro de los estratos de profundidad / Spatial distribution of fishing sites and gear implemented to capture the 'escama marina' resource within the deep strata 

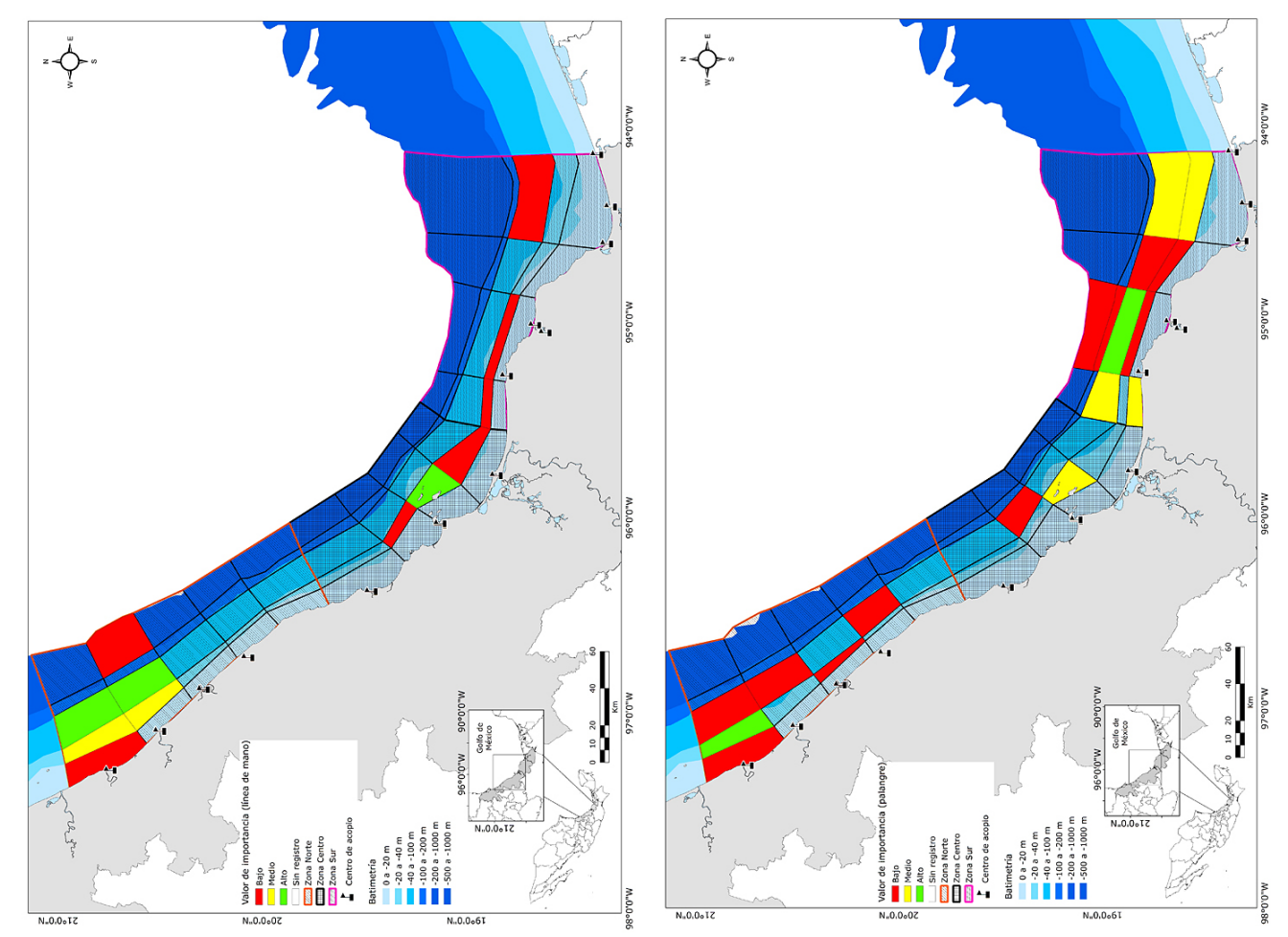

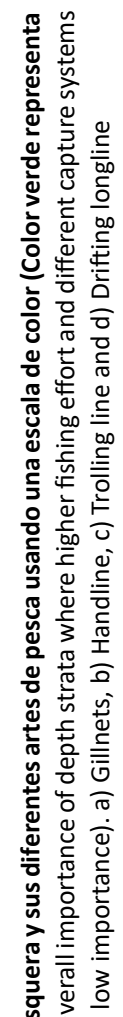

อ

ช
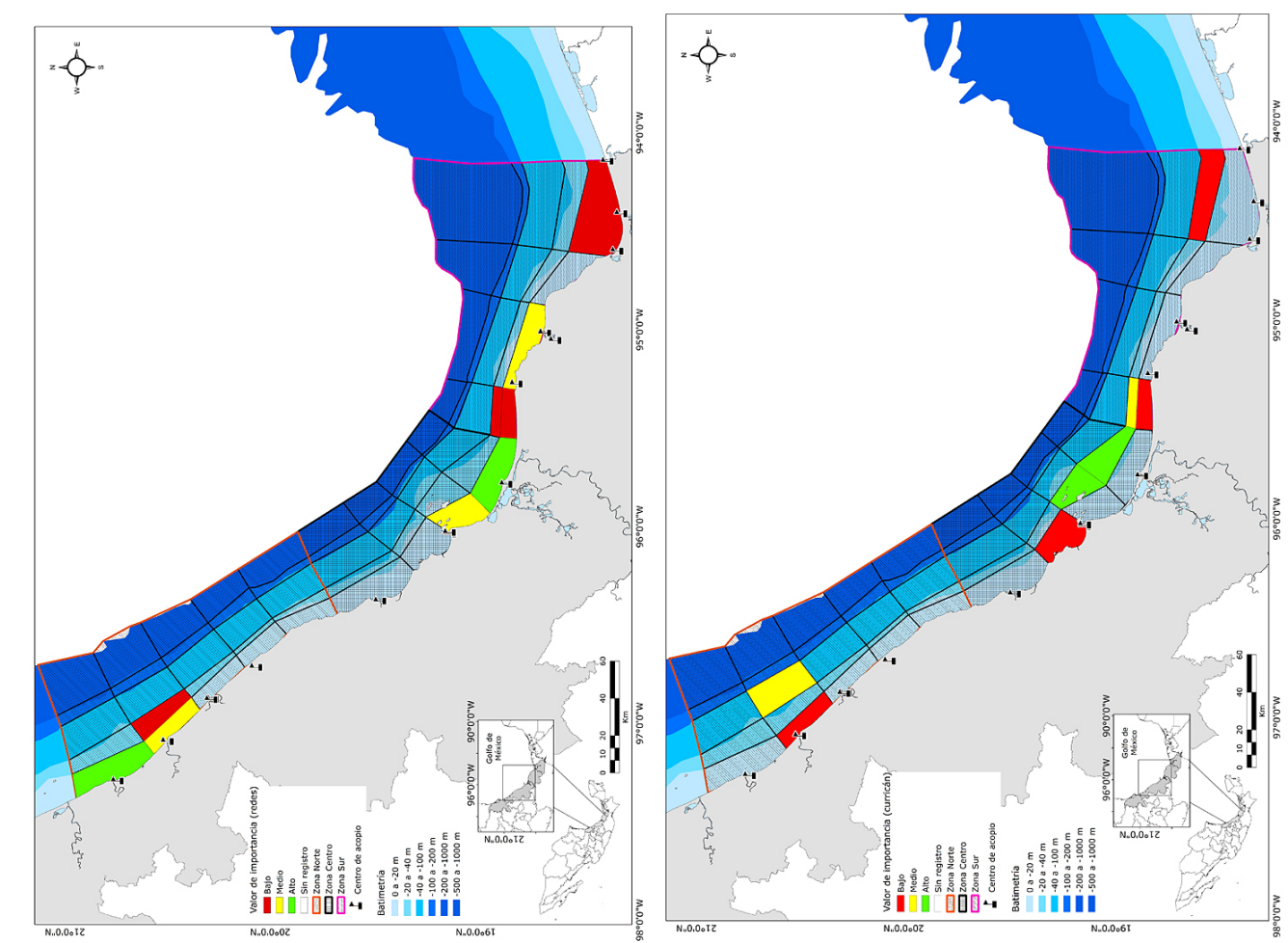

กั

ธิ 
Para la línea de mano, se implementan en zonas someras como sitios que van en rangos entre -20 a $-40 \mathrm{~m}$, aunque también se presentaron registros de este sistema de captura utilizada hasta los $-100 \mathrm{~m}$ de profundidad. Existen diferentes tipos de líneas: como cordeles, rosarios, calas y varas, cuya eficiencia varía en función del tipo de carnada, tamaño y tipo de anzuelo. Este arte de pesca se relaciona de manera directa con la captura de algunas especies de Lutjánidos, Hemúlidos y Serranidos, entre otros. En la Figura 5b, se muestran la importancia de este arte de pesca, cuyos valores de importancia más altos se encuentran en los estratos la categoría A y B (desde 0 a -40 m). En la zona norte y centro, se encontraron puntos de pesca con alta importancia en los estratos con categoría $\mathrm{C}$ ( $-40 \mathrm{a}-100 \mathrm{~m})$, así como un registro en el estrato de mayor profundidad (E: -200 a -1000 m), respectivamente.

En el caso del curricán y palangre, son artes de pesca que se implementan en zonas profundas (entre -30 a -500 m) y alejadas de la costa. Para el curricán, la eficiencia de esta arte puede variar en función del tipo de anzuelo y señuelo, la experiencia del pescador y la distribución del recurso. Esta arte de pesca es altamente selectiva, siendo las especies objetivo algunos organismos de las familias Scombridae y Carangidae. De acuerdo con la Figura 5c, los estratos en la categoría B en la zona centro, presentan alta importancia, con el mayor número de sitios de pesca a profundidades desde -20 a $-40 \mathrm{~m}$. Asimismo, se obtuvieron 2 registros de importancia media en la zona norte y centro y un registro en el estrato B, casi 10 millas náuticas frente a la comunidad de Agua Dulce, al sur del litoral Veracruzano. En comparación con el curricán, el palangre no es un arte selectivo, cuya eficiencia está determinada por la distribución de las especies, así como algunas variables físico-ambientales como son la temperatura y profundidad. La Figura 5d, muestra que los estratos de categoría $\mathrm{B}$ y $\mathrm{C}$ en la zona norte $\mathrm{y}$ sur, es donde se presentan los valores de importancia medio $\mathrm{y}$ alto, lo cual indica que varios pescadores se concentran en estas zonas de pesca utilizando este sistema de captura. En la zona sur, en el estrato de mayor profundidad (-200 a -1000 $\mathrm{m})$, se encontró un registro como sitio de pesca, lo cual se encuentra relacionado con la captura de algunas especies pelágicas de tiburones, atunes y organismos asociados a las familias Lutjanidae, Scombridae y Serranidae.

\section{DisCUSIÓN}

Considerando que las oficinas federales de pesca recaban la información (en avisos de arribo de pesca que los propios pescadores presentan) de los sitios de desembarque ubicados en sus respectivas zonas de influencia, el análisis de sus registros permite estimar la distribución de los recursos. Analizando la distribución de norte a sur, se aprecia que los grupos de escama marina, aunque se presentan en todo el litoral, son dominantes en la zona centro y sur del estado, desde Alvarado hasta Coatzacoalcos, situación inversa a los otros recursos, en donde predomina ampliamente el ostión, pues su producción es más importante en la zona norte en donde se ubican las lagunas costeras de Pueblo Viejo, Tamiahua y Tampamachoco (Quiroga-Brahms \& Romero-Hernández 2013). De acuerdo con la información obtenida de la CONAPESCA, destacan la oficina de Tuxpan, que registra el atún obtenido mediante la flota mayor palangrera cuya base se ubica en dicho puerto. La oficina de Catemaco reporta el mayor volumen de peces óseos en el estado con 4.985 t en 2010 , siendo relevante que el $60 \%$ de esta producción procede del lago, donde la tilapia representa $43 \%$, la mojarra tenguayaca el $7,2 \%$ y el topote $7,4 \%$; asimismo, es importante señalar la producción de la flota menor que opera en la zona litoral que abarca Sontecompan, Roca Partida y Balzapote entre otros sitios de desembarque de la zona costera que rodea al volcán de San Martín. La oficina de Coatzacoalcos registra la producción dulceacuícola que proviene del río con el mismo nombre y las especies marinas de la flota menor asentada en las localidades de Coatzacolacos, Villa de Allende y Barrillas, en el litoral Veracruzano (Quiroga-Brahms \& RomeroHernández 2013).

La definición de estratos es un término y un concepto considerado dentro de la zonificación marina como medio para orientar los usos humanos con el fin de optimizar la utilización de los recursos y proteger los ecosistemas marinos bajo el enfoque del ordenamiento ecológico marino (Díaz de León-Corral et al. 2006, García-Saez 2006, Rosete et al. 2006). Para esta investigación, se planteó una propuesta de zonificación del litoral Veracruzano en su porción marina, con base en los permisos revisados, la ubicación de los principales centros de acopio y la distribución espacial de actividad pesquera. De acuerdo con lo establecido por Corominas-Castiñeira (2012), este tipo de análisis permite plantear la delimitación de zonas y establecer una caracterización, diagnóstico, pronóstico y propuestas de manejo. Los objetivos en estas áreas estarían encaminados a la generación de estrategias con fines conservativos y de aprovechamiento, como sería, representar espacialmente el comportamiento de la actividad pesquera que se realiza en el litoral.

El acceso a la información a través de bases de datos oficiales del gobierno federal en combinación con los avances tecnológicos mediante el uso de Sistemas de Información Geográfica e insumos de percepción remota (Green et al. 2000, Ramírez-Rodríguez et al. 2004, Eastwood et al. 2008, Mexicano-Cíntora et al. 2009), han ayudado a mejorar el entendimiento sobre la heterogeneidad espacial en la dinámica y explotación de las poblaciones pesqueras (Martin 2004, Eastwood et al. 2008). Esto representa una oportunidad para combinar diversas bases de datos y analizar el potencial impacto de la pesca, considerando variables como son: la distribución de los recursos de interés comercial, áreas de operación de las flotas pesqueras, 
áreas de productividad biológica, temperatura del agua, corrientes marinas, tipos de fondo y accidentes topográficos. Además, se pueden considerar la distribución de lugares de desembarco y de captura (Ramírez-Rodríguez et al. 2004). Por lo anterior, la incorporación del componente espacial en el manejo de las pesquerías marinas surge como iniciativa para representar el comportamiento de la pesca de escama ribereña en el litoral Veracruzano, lo cual traería beneficios positivos a esta actividad y el ecosistema (Martin 2004, Eastwood et al. 2008).

La integración, manejo y análisis de datos utilizando los SIG, permite generar información importante sobre la dinámica poblacional y pesquera en un determinado punto geográfico (Corominas-Castiñeira 2012, Navia et al. 2015). Meaden \& Do Chi (1996) y Randall (2004) mencionan que el uso de SIG ofrecen un enorme potencial en la integración de información con fines de planeación y manejo. De acuerdo con los datos obtenidos en campo, existe una marcada heterogeneidad en cuanto al método de captura en los diferentes estratos, ya que podemos encontrar diversas artes de pesca operando en la misma profundidad sin considerar la especie objetivo, lo que infiere que la pesca sea multiespecífica y que pueda presentarse una baja selectividad en cada zona de pesca. Esta situación prevalece a lo largo del litoral del Golfo de México, sin embargo, en el área de pesca, no hay datos espaciales suficientes para conformar un análisis más estructurado con una amplia variedad de fuentes de información geográfica que permitan mejorar la calidad de los productos generados. Los resultados obtenidos han permitido identificar el comportamiento de la distribución espacial de la actividad pesquera en diferentes zonas y sistemas de captura que se utilizan, aportando las primeras bases específicas dentro del litoral Veracruzano en lo que a manejo de pesca se refiere, sin embargo, pueden ser limitados por la insuficiencia de datos que representen diferencias espaciales y temporales asociadas a la dinámica del recurso y de la flota pesquera (Ramírez-Rodríguez et al. 2004).

La necesidad de promover investigaciones que generen datos espaciales se incrementa al aumentar la demanda del uso de los recursos por parte de múltiples usuarios. La funcionalidad del SIG para evaluar patrones de comportamiento de los recursos pesqueros y sus usuarios será mayor al tener mayor disponibilidad de datos (Meaden \& Do Chi 1996, Gilliland \& Laffoley 2008). No obstante, esta investigación presenta información útil para abordar nuevas líneas, identificar huecos en el conocimiento y al mismo tiempo que pueden servir como herramientas prácticas en la discusión de talleres y reuniones de manera coordinada entre los administradores y los usuarios de los recursos pesqueros (Riolo 2006), para definir planes y estrategias de manejo sobre la dinámica de la flota de escama ribereña y su manejo potencial en el litoral Veracruzano, hasta ahora poco estudiado.
Por otro lado, el valor de importancia de los estratos de pesca permite relacionar los sitios de pesca con las artes empleadas para el recurso escama marina, y con ello muestra la tendencia de captura (Ramírez-Rodríguez et al. 2004, Salas et al. 2007, Navia et al. 2015). El arte de pesca (red) permite relacionar el tipo de especie objetivo y el patrón de comportamiento, especies como el peto $(S$. cavalla), sierra (S. maculatus), y jurel (C. hippos) mantienen un comportamiento migratorio y asociado a la costa. De acuerdo con Collette \& Nauen (1983) dentro del Golfo de México, hacia aguas mexicanas, la captura comercial para escama marina se realiza con curricán, chinchorro, almadrabas y línea de mano con cebo, y palangre pero la más destacada es la red de enmalle (Sánchez-González \& Schultz-Ruíz 1997).

El uso de las artes de pesca están directamente correlacionadas con las características batimétricas de cada sitio de pesca así como de los aspectos biológicos de las especies comerciales de escama marina. La identificación de los sitios donde operan las diferentes artes de pesca ribereña coincide con lo establecido en el manual de la FAO (Nédélec \& Prado 1990), donde menciona que las redes de enmalle son más efectivas hasta profundidades no mayores de $10 \mathrm{~m}$, dependiendo del tipo de operación de la red, siendo esta de fondo, media agua o superficie, la profundidad puede variar hasta los $20 \mathrm{~m}$, siendo esta arte muy selectiva de acuerdo al tamaño de malla; en lo que refiere a las líneas (curricán y palangre) y de mano, la profundidad de operación varía de acuerdo a su forma de uso, ya sea fija a nivel superficial, media agua o fondo alcanzando profundidades que van desde los $20 \mathrm{~m}$ hasta $\operatorname{los} 500 \mathrm{~m}$.

Como ha sido señalado con anterioridad, una importante proporción de la captura de escama marina que se realiza en México es de tipo artesanal, de la cual 98\% de esta actividad se realiza en el estado de Veracruz y se encuentra sustentada en especies migratorias que sólo pueden ser capturadas en ciertas temporadas o "corridas". Durante sus rutas migratorias, las especies marinas transitan por zonas pertenecientes a diferentes municipios, regiones o países. De esta manera, los recursos son compartidos, y dado que no reconocen barreras, la reglamentación sobre su extracción debe ser consensuada entre autoridades de diferentes municipios, acuerdos que en ocasiones no llegan a establecerse (Jiménez-Badillo 2006). Tal es el caso de algunos pescadores del municipio de Nautla que realizan sus actividades en la zona de pesca frente a Tecolutla, al norte del litoral Veracruzano, donde el esfuerzo pesquero se ha incrementado considerablemente, utilizando artes de pesca de manera poco selectivas y no respetando las áreas donde otros pescadores tienen sus zonas de pesca, ya que como lo establece el Diario Oficial de la Federación (DOF $2002)^{7}$, en los permisos de pesca de escama ribereña, la zona de pesca oficial puede ser toda la parte del Golfo de México en el estado de Veracruz. Esto ha generado en 
consecuencia, una mayor competencia entre numerosos usuarios, limitando el acceso a los recursos, ocasionando conflictos de interés por el uso de estas áreas, así como el robo de algunos sistemas de captura (DOF $2000^{10}$, Sabatini \& Sepulveda 2011), por lo que la gestión de estas pesquerías requiere una comprensión del comportamiento humano y de cómo las personas usan los recursos marinos. También requieren una estrategia diferente de manejo que va más allá de las medidas de monitoreo y vigilancia por parte de las autoridades federales (Jiménez-Badillo 2006, JiménezBadillo et al. 2008).

Hoy en día, la evaluación de las pesquerías artesanales multiespecíficas y de pequeña escala es complicada y generalmente se suscribe a la explotación de especies de importancia comercial, sin considerar su interacción con la diversidad de especies asociadas, sistemas y zonas de pesca y con la escasa infraestructura disponible (ubicación de centro de acopio y desembarco) (Salas et al. 2007, ArreguínSánchez \& Arcos-Huitrón 2011). En el litoral Veracruzano, el estudio de las zonas y áreas de pesca han sido poco estudiadas mediante el enfoque espacial, la información pesquera disponible es escasa sobre los puntos de pesca de las flota ribereñas, los datos hasta ahora obtenidos se recaban bajo encuestas y datos que se registran en los avisos de arribo (Ramírez-Rodríguez et al. 2004). De manera complementaria, el uso de información que obtienen los pescadores mediante la ayuda de los sistemas de navegación como el GPS para identificar los sitios de pesca, además de los cambios físicos y batimétricos de las zonas de captura, son raramente considerados en evaluaciones pesqueras al ser señalados como subjetivos y fraccionados (Silver \& Campbell 2005, Aswani \& Lauer 2006, Close \& Hall 2006); sin embargo, Quiroga-Brahms \& Romero-Hernández (2013) subrayan que la complejidad en el manejo pesquero, en donde intervienen aspectos biológicos, tecnológicos, sociales, culturales, ecológicos, económicos y políticos, hace necesario emplear herramientas actualizadas como los SIG y GPS, que permitan analizar e integrar regionalmente la información disponible y sus interacciones espaciales. Los resultados generados en esta investigación muestran mapas del comportamiento de la actividad pesquera de escama ribereña y posibilita que sean complementados con otras variables, así como el de actualizar la información existente.
Es evidente que las pesquerías de escama ribereña enfrentan grandes problemas de diversa índole, en general se puede considerar que la mayoría se encuentra en su máximo rendimiento o incluso en deterioro, de acuerdo con la Carta Nacional Pesquera (DOF 20185). La enorme complejidad de estas pesquerías, debido a la intrincada red de interacciones entre especies y las variaciones espaciotemporales de los recurso, clima y factores sociopolíticos, ha impedido avanzar lo suficiente en el marco normativo para su regulación, pues actualmente sólo existe una Norma Oficial Mexicana enfocada a la pesca de lisa y lebrancha (DOF 2015) ${ }^{12}$ y algunas disposiciones para el manejo de los recursos pesqueros basado en el establecimiento de épocas de veda para algunas especies, regulación de aberturas de malla y tallas mínimas de captura y en ocasiones restricción en el uso de determinados equipos de pesca (Jiménez-Badillo \& Castro-Gaspar 2007). Para fundamentar el establecimiento de estas medidas se requiere un conocimiento profundo de aspectos básicos de la biología de las especies capturadas, así como de la selectividad y coeficientes de capturabilidad de los equipos de pesca empleados; datos que para la mayoría de las pesquerías se desconocen. Además su implementación requiere de un proceso de concientización por parte de los pescadores y de un eficiente mecanismo de control y vigilancia que garanticen su cumplimiento (Jiménez-Badillo \& Castro Gaspar 2007, Quiroga-Brahms \& Romero-Hernández 2013).

En las últimas dos décadas, particularmente en el manejo de la pesca artesanal (FAO 2003, Ramírez-Rodríguez et al. 2004, Sandstrom \& Rova 2010), el uso de SIG se ha implementado como herramientas de planeación en diversas líneas estratégicas, como es el enfoque ecosistémico (Defeo 2015) para identificar y definir con mayor precisión unidades funcionales de manejo pesquero (especies objetivo y sistemas de captura), las características de la infraestructura para el manejo, procesamiento y comercialización de la captura y considerar no solo los aspectos ecológicos (Ramírez-Rodríguez et al. 2004, Quiroga-Brahms \& Romero-Hernández 2013), sino también integrar los aspectos sociales y el conocimiento de las asociaciones comunitarias del sector pesquero, los cuales adquieren un papel protagónico tanto en la gestión como en la generación de alternativas de manejo y el proceso de toma de decisiones (FAO 2018).

\footnotetext{
${ }^{12}$ DOF. 2015. Norma Oficial Mexicana NOM-016-SAG/PESC-2014, para regular la pesca de lisa y liseta o lebrancha en aguas de jurisdicción federal del Golfo de México y Mar Caribe, así como del Océano Pacífico, incluyendo el Golfo de California. Diario Oficial de la Federación, Ciudad de México, 29 de julio de 2015.
} 
El enfoque espacial propuesto en esta investigación, es una manera práctica para visualizar mediante una interfaz de SIG, el comportamiento de la pesquería de escama ribereña del litoral Veracruzano. Este estudio de carácter cualitativo permitió generar un esquema de zonificación de 3 grandes zonas de captura para la pesca ribereña, marcadas geográficamente en diferentes estratos de profundidad, de acuerdo a la forma de operación de cada arte de pesca, así como del efecto costo-beneficio que se genera para la actividad pesquera, clasificando éstas como zona norte, centro y sur. De la misma forma, se incorporó el conocimiento experto de los pescadores locales para representar la heterogeneidad espacial por las diferentes artes de pesca implementadas, identificando zonas de importancia pesquera y dos sitios de pesca con conflicto social pesquero: la localidad de Casitas con Tecolutla, y la localidad de Alvarado con Punta Puntilla, en menor conflicto esta última; debido a que en estos sitios de intersección, pescadores de las diferentes localidades compiten por disponibilidad de espacio para evitar ser invadidos dentro de sus zonas pesqueras. Por lo anterior, los resultados obtenidos podrían ser muy útiles para determinar cómo funciona la pesquería de escama ribereña y lograr una gestión localizada y centrada en las necesidades de estas localidades. Asimismo, el marco metodológico implementado en la presente investigación es el primer paso dirigido a las autoridades para dimensionar la pesca artesanal que se realiza en el litoral Veracruzano, comprender cómo opera y afecta a las comunidades pesqueras. Con esta información es posible iniciar el manejo adaptativo de los recursos pesqueros (Berkes et al. 2001, Defeo \& Castilla 2005, Breton et al. 2006) mientras se sigue avanzando en la comprensión del funcionamiento a nivel ecosistema que permita tomar decisiones más acertadas (Jiménez-Badillo \& Castro-Gaspar 2007). Los resultados de este estudio permitirán que las autoridades correspondientes al ordenamiento y administración de los recursos pesqueros en el litoral Veracruzano, consideren las zonas de pesca clasificadas, para que estas sean indicadas en los permisos de pesca según la ubicación de las localidades pesqueras. Lo anterior contribuirá a disminuir o eliminar el conflicto social que existe en los municipios de Nautla con Tecolutla y los municipios de Alvarado con Ángel R. Cabada y tener la oportunidad de considerar un ordenamiento espacial marino adecuado para la pesca ribereña en México. Por lo antes expuesto es de vital importancia considerar los resultados derivados de esta investigación para atender este tema de gran relevancia y obtener un aprovechamiento ordenado y responsable del recurso de escama ribereña en Veracruz y en el Golfo de México, a fin de seguir contando con el privilegio de contar con la riqueza de este recurso para las actuales y futuras generaciones.

\section{Agradecimientos}

Se agradece al sector pesquero por las facilidades otorgadas para el muestreo en las diferentes localidades de importancia pesquera en el litoral Veracruzano.

\section{LITERATURA CITADA}

Arreguín-Sánchez F. 2006. Pesquerías de México: (Diagnóstico y Pespectivas). En: Guzmán-Amaya P \& D FuentesCastellanos (eds). Pesca, acuacultura e investigación en México, pp. 13-36. Centro de Estudios para el Desarrollo Rural Sustentable y la Soberanía Alimentaria, Cámara de Diputados, LIX Legislatura/Congreso de la Unión, Ciudad de México.

Arreguín-Sánchez F \& E Arcos-Huitrón. 2011. La pesca en México: Estado de la explotación y uso de los ecosistemas. Hidrobiológica 21(3): 431-462.

Aswani S \& M Lauer. 2006. Incorporating fishermen's local knowledge and behavior into geographical information systems (GIS) for designing marine protected areas in Oceania. Human Organization 65: 81-102.

Beck M \& M Odaya. 2001. Ecoregional planning in marine environments: Identifying priority sites for conservation in the northern Gulf of Mexico. Aquatic Conservation: Marine and Freshwater Ecosystems 11: 235-242.

Berkes F, R Mahon, P McConney, R Pollnac \& R Pomeroy. 2001. Managing small-scale fisheries: Alternative directions and methods, 320 pp. International Development Research Centre, Ottawa.

Bezaury-Creel J. 2005. Protected areas and coastal and ocean management in Mexico. Ocean \& Coastal Management 48: 1016-1046

Breton Y, D Brown, B Davy, M Haughton \& L Ovares. 2006. Coastal resource management in the Wider Caribbean: Resilience, adaptation, and community diversity, $260 \mathrm{pp}$. Ian Randle Publishers, Kingston.

Close CH \& GB Hall. 2006. A GIS-based protocol for the collection and use of local knowledge in fisheries management planning. Journal of Environment Management 78(4): 341-352.

Collette BB \& CE Nauen. 1983. FAO Species Catalogue, Vol. 2. Scombrids of the world. An annotated and illustrated catalogue of tunas, mackerels, bonitos and related species known to date. FAO Fisheries Synopsis 125(2): 1-137.

Contreras F. 2002. Importancia de la pesca ribereña. Contactos 46: 5-14.

Corominas-Castiñeira J. 2012. El SIG como herramienta de valoración del estado de las pesquerías del Golfo de California, México. Resumen Tesis de Maestría, Universitat Politécnica de Catalunya, Barcelona, 9 pp.

Cruz-Ayala MB \& LE Igartúa-Calderón. 2006. La transformación de la legislación pesquera en México: un acercamiento en el contexto político-económico (19251992). En: Guzmán-Amaya P \& D Fuentes-Castellanos (eds). Pesca, acuacultura e investigación en México, pp. 233247. Centro de Estudios para el Desarrollo Rural Sustentable y la Soberanía Alimentaria, Cámara de Diputados, LIX Legislatura/Congreso de la Unión, Ciudad de México. 
Defeo O. 2015. Enfoque ecosistémico pesquero. Conceptos fundamentales y su aplicación en pesquerías de pequeña escala de América Latina. FAO Documento Técnico de Pesca y Acuacultura 592: 1-94.

Defeo O \& JC Castilla. 2005. More than one bag for the world fishery crisis and keys for co-management successes in selected artisanal Latin American shellfisheries. Reviews in Fish Biology and Fisheries 15(3): 265-283.

Del Rincón D, J Arnal, A Latorre \& A Sans. 1995. Técnicas de investigación en ciencias sociales, 427 pp. Editorial Dykinson, Madrid.

Díaz de León-Corral A, J Fernández, P Álvarez-Torres, O Ramírez-Flores \& LG López-Lemus. 2004. La sustentabilidad de las pesquerías del Golfo de México. En: Caso M, I Pisanty \& E Ezcurra (eds). Diagnóstico ambiental del Golfo de México. Volumen 2, pp. 725-753. Instituto Nacional de Ecología, México D.F.

Díaz de León-Corral A, P Álvarez-Torres \& O IglesiasBarrón. 2006. Experiencias globales de clasificación y ejercicios de zonificación marina. En: Córdova y Vázquez A, F Rosete-Vergés, G Enríquez-Hernández \& B Hernández de la Torre (comp). Ordenamiento ecológico marino. Visión integrada de la regionalización, pp. 21-41. Instituto Nacional de Ecología, México D.F.

Dipboye R. 1994. Structured and unstructured selection interview: beyond the job-fit model. Research in Personnel and Human Resources Management 12: 79-123.

Eastwood P, G Meaden, T Nishida \& S Rogers. 2008. Understanding and managing marine fisheries with the aid of a digital map. In: Payne A (ed). Advances in Fisheries Science. 50 years on from Beverton and Holt, pp. 85-103. Blackwell Publishing, Iowa.

Espinoza-Tenorio A, M Moreno-Báez, D Pech, GJ VillalobosZapata, L Vidal-Hernández, J Ramos-Miranda, M Mendoza-Carranza, JA Zepeda-Domínguez, G AlcaláMoya, JC Pérez-Jiménez, F Rosete, C León \& I Espejel. 2014. El ordenamiento ecológico marino en México: un reto y una invitación al quehacer científico. Latin American Journal of Aquatic Research 42(3): 386-400.

FAO. 1996. Precautionary approach to capture fisheries and species introductions. FAO Technical Guidelines for Responsible Fisheries 2: 1-54.

FAO. 2003. Geographic information systems in fisheries management and planning. FAO Fisheries Technical Paper 449: 1-162.

FAO. 2018. Cumplir los objetivos de desarrollo sostenible, $250 \mathrm{pp}$. Organización de las Naciones Unidas para la Alimentación y la Agricultura. Departamento de Pesca y Acuicultura de la FAO, Roma.

FAO \& SAGARPA. 2014. Diagnóstico del sector rural y pesquero de México 2012, 45 pp. Organización de las Naciones Unidas para la Alimentación y la Agricultura y Secretaría de Agricultura, Desarrollo Rural, Pesca y Alimentación, Ciudad de México.

García S. 1994. The precautionary principle: its implications in capture fisheries management. Ocean \& Coastal Management 22: 99-125.

García-Sáez C. 2006. Caracterización de la distribución de especies de importancia biológica en el ordenamiento ecológico marino: Evaluación de metodologías existentes y propuesta de agenda de investigación. En: Córdova y Vásquez A, F Rosete-Vergés, G Enríquez-Hernández \& B Hernández de la Torre (comp). Ordenamiento ecológico marino. Visión integrada de la regionalización, pp. 87-105. Instituto Nacional de Ecología, México D.F.

González-Pedrero J. 2006. Desempeño nacional e internacional. Política, regulación, control y apoyo al sector. En: GuzmánAmaya P \& D Fuentes-Castellanos (eds). Pesca, acuacultura e investigación en México, pp. 203-211. Centro de Estudios para el Desarrollo Rural Sustentable y la Soberanía Alimentaria, Cámara de Diputados, LIX Legislatura/ Congreso de la Unión, Ciudad de México.

Green E, P Mumby, A Edwards \& C Clark. 2000. Remote sensing. Handbook for tropical coastal management. Coastal Management Sourcebooks 3: 1-316. UNESCO, Paris.

Gilliland P \& D Laffoley. 2008. Key elements and steps in the process of developing ecosystem-based Marine Spatial Planning. Marine Policy 32: 787-796.

Ibáñez de la Calle M, M Becerra Pérez \& G Brachet-Barro. 2004. Cuotas individuales transferibles: Una alternativa para resolver la problemática de las pesquerías en México. Gaceta Ecológica, Instituto Nacional de Ecología 70: 31-43.

Jiménez-Badillo ML. 2006. Caracterización de la pesca en la zona costera Veracruzana. En: Moreno-Casasola P (ed). Estrategias para el manejo integral de la zona costera: un enfoque municipal, pp. 265-295. Instituto de Ecología A.C. y Comisión Nacional de Áreas Naturales Protegidas, SEMARNAT, Xalapa.

Jiménez-Badillo ML \& LG Castro-Gaspar. 2007. Pesca artesanal en el Parque Nacional Sistema Arrecifal Veracruzano. En: Granados-Barba A, LG Abarca-Arenas \& JM Vargas-Hernández (eds). Investigaciones científicas en el Sistema Arrecifal Veracruzano, pp. 221-240. Universidad Autónoma de Campeche, Campeche.

Jiménez-Badillo ML, V Arenas-Fuentes \& H Pérez-España. 2008. The Conservation-Exploitation Paradox in a Mexican Coral Reef Protected Area. American Fisheries Society Symposium 49: 587-595.

Lucca-Irizarry N \& R Berríos-Rivera. 2009. Investigación cualitativa: fundamentos, diseños y estrategias, $623 \mathrm{pp}$. Ediciones SM, Cataño.

Martin K. 2004. GIS in marine fisheries science and decision making. In: Fisher W (ed). Geographic Information Systems in fisheries, pp. 237-258. American Fisheries Society, Maryland.

Meaden GJ \& T Do Chi. 1996. Geographical information systems: applications to machine fisheries. FAO Fisheries Technical Paper 356: 1-335.

Mexicano-Cíntora G, MA Liceaga-Correa \& S Salas. 2009. Uso de sistemas de información geográfica en pesquerías: la pesca en Yucatán, al sur del Golfo de México. Universidad y Ciencia 25(1): 23-28.

Muñoz-Villanueva J \& RE Acosta-Jenkins. 2006. El papel del Estado con relación al sector. En: Guzmán-Amaya P \& D Fuentes-Castellanos (eds). Pesca, acuacultura e investigación en México, pp. 217-225. Centro de Estudios para el Desarrollo Rural Sustentable y la Soberanía Alimentaria, Cámara de Diputados, LIX Legislatura/Congreso de la Unión, Ciudad de México. 
Navia AF, P Mejía-Falla, JA Caicedo \& AT López. 2015. Caracterización espacial de la pesca artesanal en la subregión Sanquianga-Gorgona, $9 \mathrm{pp}$. Informe Técnico Final - Equipo de trabajo SQUALUS-WWF, Fundación Colombiana para la Investigación y Conservación de Tiburones y Rayas SQUALUS.

Nédélec C \& J Prado. 1990. Definition and classification of fishing gear categories. FAO Fisheries Technical Paper 222: $1-92$

Ólafsdóttir H \& R Rafnsson. 2012. Sectores basados en recursos biológicos. Capítulo 6: Pesca. En: Dufresne C (ed). Enciclopedia de Salud y seguridad en el trabajo, pp. 1-21. Ministerio de Trabajo y Asuntos Sociales Subdirección General de Publicaciones, Madrid.

Quiroga-Brahms C \& E Romero-Hernández. 2013. Estado actual de las pesquerías de peces en el estado de Veracruz. En: Aldana-Aranda D, M Enríquez Díaz \& V Elías (eds). Manejo de los recursos pesqueros de la cuenca del Golfo de México y del Mar Caribe, pp. 258-285. Universidad Veracruzana, Xalapa.

Ramírez-Rodríguez M, C López-Ferreira \& A HernándezHerrera. 2004. Desarrollo de un sistema de información geográfico como apoyo para la administración de la pesca artesanal en México. En: Salas S, MA Cabrera, J Ramos, D Flores \& J Sánchez (eds). Memorias primera conferencia de pesquerías costeras en América Latina y el Caribe. Evaluando, manejando y balanceando acciones, pp. 200207. Mérida.

Randall MP. 2004. Possible solutions to some challenges facing fisheries scientists and managers. ICES Journal of Marine Science 61: 1331-1343.

Reyna-González, PC, J Bello-Pineda, L Ortíz-Lozano, H Pérez-España, P Arceo \& J Brenner. 2014. Incorporating expert knowledge for development spatial modeling in assessing ecosystem services provided by coral reefs: A tool for decision-making. Revista de Biología Marina y Oceanografía 49(2): 279-292.

Riolo F. 2006. A geographic information system for fisheries management in American Samoa. Environment Modelling Software 21(7): 1025-1041.

Rosete F, G Enríquez \& A Córdova. 2006. Introducción. En: Córdova y Vásquez A, F Rosete-Vergés, G EnríquezHernández \& B Hernández de la Torre (comp). Ordenamiento ecológico marino. Visión integrada de la regionalización, pp. 13-19. Instituto Nacional de Ecología, México D.F.
Ruíz-Moreno R \& C Mérigo-Orellana. 2006. La industria pesquera. En: Guzmán-Amaya P \& D Fuentes-Castellanos (eds). Pesca, acuacultura e investigación en México, pp. 227232. Centro de Estudios para el Desarrollo Rural Sustentable y la Soberanía Alimentaria, Cámara de Diputados, LIX Legislatura/Congreso de la Unión, Ciudad de México.

Sabatini F \& C Sepúlveda. 1997. Conflictos ambientales. Entre la globalización y la sociedad civil, 383 pp. Publicaciones CIPMA, California.

Salas S, R Chuenpagdee, JC Seijo \& A Charles. 2007. Challenges in the assessment and management of small-scale fisheries in Latin American and the Caribbean. Fisheries Research 87: 5-16.

Sánchez-González S \& LE Schultz-Ruíz. 1997. Descripción de la pesquería de sierra y peto. En: Flores-Hernández D, P Sánchez-Gil, JC Seijo \& F Arreguín-Sánchez (eds). Análisis y diagnóstico de los recursos pesqueros críticos del Golfo de México, pp. 227-248. Universidad Autónoma de Campeche, Campeche.

Sandstrom A \& C Rova. 2010. Adaptive co-management networks: a comparative analysis of two fishery conservation areas in Sweden. Ecology and Society 15(3): 1-14.

Silver JJ \& LM Campbell. 2005. Fisher participation in research: Dilemmas with the use of fisher. Ocean and Coastal Management 48: 721-741.

TNC. 2005. Planificación para la conservación de áreas. Desarrollo de estrategias, ejecución de acciones y medidas de éxitos en cualquier escala, 23 pp. The Nature Conservancy, Texas.

Valles M. 1997. Técnicas cualitativas de investigación social: reflexión metodológica y práctica profesional, $430 \mathrm{pp}$. Editorial Síntesis, Madrid.

Vázquez-León CI. 2006. Desarrollo, sustentabilidad y pobreza. Perspectivas de índole socioeconómica en comunidades dedicadas a la pesca ribereña. En: Guzmán-Amaya P \& D Fuentes-Castellanos (eds). Pesca, acuacultura e investigación en México, pp. 171-189. Centro de Estudios para el Desarrollo Rural Sustentable y la Soberanía Alimentaria, Cámara de Diputados, LIX Legislatura/Congreso de la Unión, Ciudad de México. 